\title{
L1CAM as a Negative Prognostic Factor in Endometrioid Endometrial Adenocarcinoma FIGO Stage IA-IB
}

\author{
JAROSLAV KLAT $^{1}$, ALES MLADENKA ${ }^{1}$, JANA DVORACKOVA $^{2}$, SYLVA BAJSOVA $^{1}$ and ONDREJ SIMETKA ${ }^{1}$ \\ ${ }^{1}$ Department of Obstetrics and Gynecology, University Hospital Ostrava, Ostrava Poruba, Czech Republic; \\ ${ }^{2}$ Institute of Pathology, University Hospital Ostrava, Ostrava Poruba, Czech Republic
}

\begin{abstract}
Aims: In this study, we aimed to investigate how positivity for L1 cell adhesion molecule (L1CAM) was associated with outcome and relapse pattern in patients with Fédération Internationale de Gynécologie et d'Obstétrique (FIGO) stage IA-IB endometrial cancer. Materials and Methods: This retrospective study included 358 patients who underwent surgical treatment for endometrial carcinoma. Tumor samples from 312 patients (87.2\%) were available for LICAM analysis by immunohistochemistry. Results: Of the 312 tumor samples analyzed, 93 (29.8\%) were L1CAMpositive. LICAM positivity was significantly more common in grade 3 compared to grade 1-2 carcinomas $(p=0.02)$. Patients with L1CAM positivity more commonly experienced disease progression. Distant metastasis was significantly associated with LICAM positivity ( $p=0.01)$. Progression-free interval and overall survival did not significantly differ between LICAM-positive and L1CAM-negative cases. Conclusion: LICAM is a promising independent prognostic marker associated with aggressive tumor behavior and recurrence risk, but not with overall survival.
\end{abstract}

Endometrial cancer (EC) is the sixth most common malignancy in women worldwide (1). Based on clinical and histological features, ECs are divided into type I and type II tumors. Type I tumors constitute $85 \%$ of ECs, are most commonly found in younger women, and develop from a precursor lesion of atypical hyperplasia. These tumors tend to be endometrioid ECs (EECs), are usually welldifferentiated, and typically exhibit minimal myometrial invasion; therefore, type I ECs often have a favorable outcome. Type II tumors account for a small percentage of ECs, are commonly found in older patients, and frequently

Correspondence to: Jaroslav Klat, MD, Ph.D., Department of Obstetrics and Gynecology, University Hospital Ostrava, 17.listopadu 1790, Ostrava Poruba, 708 52, Czech Republic. E-mail: jaroslav.klat@fno.cz

Key Words: Endometrial cancer, L1CAM, early stage, recurrence. develop in the setting of an atrophic endometrium. The type II category includes serous tumors, clear cell tumors and, possibly, grade 3 EECs. Notably, translational science data support the differentiation of these two groups at the molecular level. Molecular analysis further identifies four categories of EECs: polymerase epsilon ultramutated, microsatellite instability hypermutated, copy-number low, and copy-number high (2). Current guidelines stratify patients with EC based on tumor type, stage, depth of myometrial invasion, and presence of lymphovascular space invasion, delineating four risk groups: Low, intermediate, high-intermediate and high (3).

Although EEC type I tumors generally have a good prognosis, relapse occurs in some patients with low and intermediate risk. One current challenge is to understand the tumor biology as it relates to predicting recurrence and survival. Several recent studies show that positivity for L1 cell adhesion molecule (L1CAM) is strongly associated with more aggressive EEC behavior (4), risk of recurrence, and poor survival (5-10). L1CAM belongs to the immunoglobulin superfamily of cell adhesion molecules. Initially identified in the nervous system, L1CAM plays key roles in nervous system development, neuronal migration, neurite outgrowth on Schwann cells, and neurite fasciculation and myelination (11). In cancer cells, L1CAM promotes cell proliferation, migration, invasion, and metastasis. Its expression is associated with tumor progression in many types of cancers, including colorectal, gastric, renal, breast cancer and melanoma (12-18).

In the present study, we aimed to identify the clinicopathological features of L1CAM-positive Fédération Internationale de Gynécologie et d'Obstétrique (FIGO) stage IA-IB EC in patients, and to confirm the prognostic value of L1CAM.

\section{Materials and Methods}

Study cohort. This study enrolled patients who underwent primary surgical treatment for endometrial carcinoma at the University Hospital Ostrava between 2007-2015. Cases with histologically confirmed endometrioid adenocarcinoma, and with available complete surgical and follow-up data, were eligible for study 
inclusion. Patients with other types of adenocarcinoma (clear cell, serous, etc.) or with incomplete data were excluded from the study. A totaI of 358 eligible patients were identified but it was not possible to analyze histopathological samples from 46 of these cases; thus, the final number of patients included was 312. Clinical data were collected from the secure hospital database and from the patients' charts. Data were recorded during the initial hospital stay and follow-up visits. The follow-up period was at least 2 years. Written informed consent was obtained from all patients, and this study was approved by the Local Ethic Committee (approval number: 353/2015).

Disease stage was determined using the FIGO 2009 classification all cases recorded before 2009 were reclassified according to this norm (19). We did not perform a specialized gynecopathological case review to exclude potential mixed-type carcinomas containing components other than endometrioid type. Risk for recurrence was classified following published guidelines (3). Surgery and adjuvant treatment were performed according to the preoperative and perioperative (frozen section) staging procedure. In general, surgery comprised extrafascial hysterectomy and bilateral salpingooophorectomy alone or with pelvic/para-aortic lymphadenectomy. Surgery was adapted to risk and performance status.

Immunohistochemistry. The immunohistochemical procedure used was essentially that originally described by Bosse et al. (5). Formalin-fixed paraffin-embedded tissue blocks were cut into $4-\mu \mathrm{m}$ slices and placed on superfrost microscope slides. Immunohistochemistry was then performed using a Ventana Discovery automated immunostaining system (Ventana Medical Systems, Tucson, AZ, USA). Briefly, after deparaffinization with an inorganic buffer and rehydration, antigen retrieval was achieved by incubating the slides in Cell Conditioning Solution 1 (CC1; Ventana Medical Systems). The primary L1 antibody (CD171) (BioLegend, Dedham, MA, USA) was then applied at a 1:40 dilution, followed by a 32-min incubation. Next, diaminobenzidne- and horseradish peroxidasecontaining detection kit was used (iView Universal DAB detection Kit; Ventana Medical Systems), and performed counterstaining with hematoxylin and bluing reagents.

Immunohistochemical evaluation of L1CAM and scoring were performed by two experienced pathologists dedicated for this study. Only unequivocal membranous staining was considered specific. A case was classified as L1CAM-positive if over $10 \%$ of tumor cells were scored positive (10).

Statistical analyses. Statistical analyses were performed using R (R Foundation for Statistical Computing, Vienna, Austria; https:// www.Rproject.org/). Between-group differences were analyzed using the chisquared and Fisher's exact probability tests. A $p$-value of less than 0.05 was considered to indicate a statistically significant difference.

\section{Results}

Of the 312 analyzed FIGO stage I endometrioid endometrial carcinomas, 93 (29.8\%) were L1CAM-positive. Patients with L1CAM positivity were more likely to experience disease progression. However, progression-free interval (PFI) and overall survival did not significantly differ between L1CAMpositive and L1CAM-negative cases. With regards to type of disease progression, L1CAM positivity was significantly associated with distant metastasis $(p=0.01)$. L1CAM positivity was significantly less common in grade 1-2 carcinomas compared to grade 3 carcinomas $(p=0.02)$. Only a small number of patients $(n=13)$ had grade 3 tumors, and these cases did not show disease progression.

The median age at diagnosis for the entire study population was 63.4 years (range $=27-89$ years). L1CAM status was not related to patient age at diagnosis. Diabetes mellitus and obesity, which are classical epidemiological risk factors for type I EC, were not associated with L1CAM status or disease progression. The mean body mass index was $32.4 \mathrm{~kg} / \mathrm{m}^{2}$ in the L1CAM-negative group, and $31.6 \mathrm{~kg} / \mathrm{m}^{2}$ in the L1CAMpositive group $(p=0.17)$. A total of 28 patients died during follow-up, including 16 deaths due to disease progression (Table I).

\section{Discussion}

The present study was designed to evaluate the association of L1CAM positivity with outcome in patients with FIGO IA-B stage EC, and to compare relapse patterns between L1CAM-positive and L1CAM-negative carcinomas.

Recent studies show that L1CAM positivity in EC is strongly associated with more aggressive tumor behavior, greater recurrence risk, and worse overall survival (5-10). L1CAM expression rates are reportedly higher in nonendometrioid cancer than in endometrioid cancer, and prior reports describe L1CAM expression rates ranging from 7 $26.6 \%$ in endometrioid cancer $(5-10,20)$. While previous studies have excluded non-endometrioid histotypes, we did not perform a retrospective specialized gynecopathological case review to exclude potential mixed-type carcinomas containing components. Thus, our sample better reflects cases in daily clinical practice, and we found a L1CAM expression rate of $29.8 \%$, which is the highest rate in the published literature as far as we are aware.

Zeimet et al. performed the largest multicenter cohort study, and reported L1CAM to be the strongest prognostic factor in FIGO stage I type I ECs (10). Their results showed L1CAM-positive cancer to be associated with a shorter disease-free interval and worse overall survival. Pasanen et al. demonstrated that L1CAM positivity predicted poor disease-specific survival in endometrioid carcinoma, but not in non-endometrioid cancer (8). Based on these findings, Kommoss et al. proposed that the definition of the low-risk category should be limited to L1CAM-negative EC (21). In contrast, our present results showed that disease-free interval and overall survival did not significantly differ between the L1CAM-negative and L1CAM-positive groups, which is in agreement with the findings of Dellinger et al. (6) and Smogeli et al. (4). We also found that L1CAM status was not related to diabetes mellitus or obesity, which are classical epidemiological risk factors for type $\operatorname{I~EC~}(8,10)$. 
Table I. Patient characteristics.

\begin{tabular}{|c|c|c|c|c|}
\hline \multirow[b]{2}{*}{ Characteristic } & \multicolumn{2}{|c|}{ L1CAM, N (\%) } & \multirow[b]{2}{*}{ Total, N (\%) } & \multirow[b]{2}{*}{$p$-Value } \\
\hline & Negative & Positive) & & \\
\hline Patients, n (\%) & $219(70.2)$ & $93(29.8)$ & $312(100)$ & - \\
\hline \multicolumn{5}{|l|}{ Age, years } \\
\hline Mean (range) & $63.0(27-89)$ & $64.4(44-83)$ & $63.4(27-89)$ & 0.30 \\
\hline Adjuvant therapy, n (\%) & $49(22.4)$ & $22(23.7)$ & $71(22.8)$ & 0.80 \\
\hline Pelvic lymphadenectomy, n (\%)* & $91(41.6)$ & $28(30.1)$ & $120(38.3)$ & 0.056 \\
\hline \multicolumn{5}{|l|}{ Disease characteristics } \\
\hline \multicolumn{5}{|l|}{ FIGO stage, n (\%) } \\
\hline IA & $162(74.0)$ & $72(77.4)$ & $234(75.0)$ & 0.52 \\
\hline IB & $57(26.0)$ & $21(22.6)$ & $78(25.0)$ & \\
\hline \multicolumn{5}{|l|}{ Grade $1, \mathrm{n}(\%)$} \\
\hline 1 & $150(68.5)$ & $54(58.1)$ & $204(65.4)$ & 0.02 \\
\hline 2 & $64(29.2)$ & $31(33.3)$ & $95(30.4)$ & \\
\hline 3 & $5(2.3)$ & $8(8.6)$ & $13(4.2)$ & \\
\hline Disease progression, n (\%) & $10(4.6)$ & $10(10.8)$ & $20(6.4)$ & 0.04 \\
\hline \multicolumn{5}{|l|}{ Progression-free interval, months } \\
\hline Mean (range) & $24.1(4-73)$ & $24.8(1-50)$ & $24.5(1-73)$ & 0.93 \\
\hline Death, n $(\%)$ & $9(4.1)$ & $7(7.5)$ & $16(5.1)$ & 0.25 \\
\hline \multicolumn{5}{|l|}{ Overall survival, months } \\
\hline Mean (range) & $44.6(24-80)$ & $53.1(28-80)$ & $48.3(24-80)$ & 0.42 \\
\hline
\end{tabular}

L1CAM: L1 cell adhesion molecule; FIGO: Fédération Internationale de Gynécologie et d'Obstétrique. *With/without para-aortic lymphadenectomy.

In Zeimet et al.'s study, 117 patients $(11.5 \%)$ experienced recurrence over a median follow-up of 54.3 months (10). Recurrence was detected in $51.4 \%$ of L1CAM-positive tumors, and $2.9 \%$ of L1CAM-negative tumors. In our study, recurrence was detected in only $6.4 \%$ of patients with stage I EC, with recurrence rates of $4.6 \%$ among L1CAMnegative tumors and $10.8 \%$ among L1CAM-positive tumors $(p=0.04)$.

Zeimet et al. also found that extra-abdominal relapses were more frequent in L1CAM-positive (13.2\%) than L1CAM-negative (1.9\%) FIGO stage I endometrioid carcinomas $(p<0.0001)$. In contrast, other site-specific relapses showed no association with L1CAM status (10). Bosse et al.'s study revealed that L1CAM positivity is significantly correlated with risk of distant recurrence (hazard ratio=5.1) but not with vaginal relapses (5). Similarly, other authors reported a greater frequency of distant relapses in L1CAM-positive cases $(4,8)$. In agreement with these findings, our present data regarding type of disease progression indicated that only distant metastasis was significantly associated with L1CAM positivity. One strength of our present study was that it included an unselected cohort of patients who underwent treatment at a tertiary care center, following a standardized surgical technique and regular follow-up. A weakness of our study was its retrospective design and the small number of recurrences. As in all studies focusing on cancer with limited malignant potential, we faced the statistical dilemma of having to calculate survival rates based on a limited number of disease-specific events during follow-up.

In conclusion, we herein report that L1CAM is a promising independent prognostic marker that was strongly associated with more aggressive cancer behavior and recurrence risk, but not with overall survival. Prospective trials are needed to evaluate the clinical significance of L1CAM for risk assessment among low-risk patients, and to guide potential alterations of surgical and adjuvant treatment strategies.

\section{Acknowledgements}

This study was supported by institutional grant no. 2 RVOFNOs/2015.

\section{References}

1 Bray F, Ferlay J, Soerjomataram I, Siegel RL, Torre LA and Jemal A: Global cancer statistics 2018: GLOBOCAN estimates of incidence and mortality worldwide for 36 cancers in 185 countries. CA Cancer J Clin 68: 394-424, 2018.

2 Kandoth C, Schultz N, Cherniack AD, Akbani R, Liu Y, Shen H, Robertson AG, Pashtan I, Shen R, Benz CC, Yau C, Liard PW, Ding L, Zhang W, Mills GB, Kucherlapati R, Mardis ER and Levine DA: Integrated genomic characterization of endometrial carcinoma. Nature 497: 67-73, 2013. 
3 Colombo N, Creutzberg C, Amant F, Bosse T, Gonzáles-Martin A, Ledermann J, Marth C, Nout R, Querleu D, Mirza MR and Sessa C: ESMO-ESGO-ESTRO consensus conference on endometrial cancer, diagnosis, treatment and follow-up. Int J Gynecol Cancer 26: 2-30, 2016.

4 Smogeli E, Davidson B, Cvancarova M, Holth A, Katz B, Risberg B, Kristensen $\mathrm{G}$ and Lindemann $\mathrm{K}$ : L1CAM as a prognostic marker in stage I endometrial cancer: a validation study. BMC Cancer 16: 596-604, 2016.

5 Bosse T, Nout RA, Stello E, Dreef E, Nijman HW, JürgenliemSchulz IM, Jobsen JJ, Creutzberg CL and Smit VT: L1 cell adhesion molecule is a strong predictor for distant recurrence and overall survival in early stage endometrial cancer: Pooled PORTEC trial reset. E J Cancer 50: 2602-2610, 2014.

6 Dellinger TH, Smith DD, Ouyang Ch, Warden CD, Willims JC and Han ES: L1CAM is an independent predictor of poor survival in endometrial cancer - An analysis of The Cancer Genome Atlas (TGCA). Gynecol Oncol 141: 336-340, 2016.

7 Geels YP, Pijnenborg JM., Gordon BB, Vogel M, Altevogt P, Masadah R, Bulten J, van Kempen LC and Massuger LF: L1CAM expression is related to non-endometrioid histology, and prognostic for poor outcome in endometroid endometrial carcinoma. Pathol Oncol Res 22: 863-868, 2016.

8 Pasanen A, Tuomi T, Isola J, Staff S, Bützow R and Loukovaara M: L1 cell adhesion molecule as a predictor of disease-specific survival and patterns of relapse in endometrial cancer. Int J Gynecol Cancer 26: 1465-1471, 2016.

9 Van der Putten LJM, Visser NCM, van de Vijver K, Santacana M, Bronsert P, Bulten J, Hirschfeld M, Colas E, Gil-Moreno A, Garcia A, Mancebo G, Alameda F, Trovik J, Kopperud RK, Huvila J, Schrauwen S, Koskas M, Walker F, Weinberger V, Minar L, Jandakova E, Snijders MP, van der Berg-van Erp S, Matias-Guiu X, Salvesen HB, Amant F, Massuger LF and Pijneborg JM: L1CAM expression in endometrial carcinomas: An ENITEC collaboration study. B J Cancer 115: 716-724, 2016.

10 Zeimet AG, Reimer D, Huszar M, Winterhoff B, Puistola U, Azim SA, Müller-Holzner E, Ben-Arie A, van Kempen LC, Petru E, Jahn S, Geels YP, Massuger LF, Amant F, Polterauer S, Lappi-Blanco E, Bulten J, Mauter A, Tanouye S, oppelt P, StrohWeigert S, Reinthaller A, Mariani A, Hackl W, Netzer M, Schirmer U, Vergote I, Altevogt P, Marth C and Fogel M: L1CAM in early stage type I endometrial cancer. Result of a large multicenter evaluation. J National Cancer Institute 105: 1142-1150, 2013.

11 Brummendorf T and Rathjen FG: Cell adhesion molecules L1: immunoglobulin superfamily. Protein Profile 2: 963-1108, 1995.
12 Altevogt P, Doberstein K and Fogel M: L1CAM in human cancer. Int J Cancer 138: 1565-76, 2016.

13 Fogel M, Gutwein P, Mechtersheimer S, Riedle S, Stoeck A, Smirnov A, Edler L, Ben-Arie A, Huszar M and Altevogt P: L1 expression as a predictor of progression and survival of patients with uterine and ovarian carcinomas. Lancet 362: 869-875, 2003.

14 Fogel M, Mechtersheimer S, Huszar M, Smirnov A, Abu-Dahi A, Tilgen W, Reichrath J, Georg T, Altevogt P and Gutwein P: L1 adhesion molecule (CD171) in development and progression of human malignant melanoma. Cancer Lett 189: 237-247, 2003.

15 Boo YJ, Park JM, Kim J, Chae YS, Min BW, Um JW and Moon HY: L1 expression as a marker for poor prognosis, tumor progression, and short survival in patients with colorectal cancer. Ann Surg Oncol 14: 1703-1711, 2007.

16 Allory Y, Matsuoka Y, Bazille C, Christensen EI, Ronco P and Debiec H: The L1 cell adhesion molecule is induced in renal cancer cells and correlates with metastasis in clear cell carcinoma. Clin Cancer Res 11: 1190-1197, 2005.

17 Kodera Y, Nakanishi H, Ito S, Misawa K, Ito Y, Nakayama G, Koike M, Fujiwara M, Yamamura Y and Nakao A: Expression of L1 cell adhesion molecule is a significant prognostic factor in pT3-stage gastric cancer. Anticancer Res 29: 4033-4039, 2009.

18 Schröder C, Schumacher U, Fogel M, Feuerhake F, Müller V, Wirtz RM, Altevogt P, Krenkel S, Jänicke F and Milde-Langosch $\mathrm{K}$ : Expression and diagnostic value of L1-CAM in breast cancer. Oncol Rep 22: 1109-1117, 2009.

19 Pecorelli S: Revised FIGO staging for carcinoma of the vulva, cervix and endometrium. Int J Gynecol Obstet 105: 103-104, 2009.

20 Pasanen A, Loukovaara M, Tuomi T and Bützow R: Preoperative risk stratification of endometrial carcinoma: L1CAM as a biomarker. Int J Gynecol Cancer 27: 1318-1324, 2017.

21 Kommoss F, Kommoss F, Grevenkamp F, Bunz AK, Taran FA, Fenf F, Brucker SY, Wallwiener D, Schönfish B, Greif K, Lax S, Staebler A and Kommos S: L1CAM: Amending the 'low-risk' category in endometrial carcinoma. J Cancer Res Clin Oncol 143: 255-262, 2017. 